\title{
Decomposition of Many-Body Schrödinger Operators
}

\author{
E. Balslev \\ Matematisk Institut, Aarhus Universitet, DK-8000 Aarhus C, Denmark
}

\begin{abstract}
The complex-dilated many-body Schrödinger operator $H(z)$ is decomposed on invariant subspaces associated with the "cuts" $\left\{\mu+z^{-2} R^{+}\right\}$, where $\mu$ is any threshold, and isolated spectral points. The interactions are dilation-analytic multiplicative two-body potentials, decaying as $r^{-1+\varepsilon}$ at $r=0$ and as $r^{-\varepsilon}$ at $r=\infty$.
\end{abstract}

\section{Introduction}

The non-relativistic quantum mechanical many-body problem has been the object of several mathematical investigations during recent years. One of the central problems is that of asymptotic completeness for systems with multichannel scattering. Since the work of Faddeev [4] on the 3-body problem new major steps have been taken by Ginibre and Moulin [5] and Thomas [11] who have independently generalized Faddeev's result in the 3-body case and at the same time simplified the proofs, utilizing different, though related Hilbert space methods. Another important work is due to Sigal [9] who has extended Faddeev's result to the $n$-body case under essentially the same assumptions and utilizing the same mathematical tools, working with Banach spaces of Hölder-continuous functions.

A different approach to the $n$-body problem was introduced by the author and Combes [3] and independently by van Winter [12]. This may be called the analytic theory of many-body Schrödinger operators, with the dilation-analytic version of [3] and the complex dynamical variables version of [12]. The basic feature of this theory is the construction of a selfadjoint analytic family of operators $H(z)$ in an angle $S_{a}=\left\{z=\varrho e^{i \varphi}|\varrho>0| \varphi \mid<a\right\}$, such that $H(1)=H$. The operators $\mathrm{H}\left(\varrho e^{i \varphi}\right), \varphi \neq 0$, although non-selfadjoint, are more accessible for analysis than $H$ itself, whose properties can then be derived from those of $H\left(e^{i \varphi}\right)$ by letting $\varphi \rightarrow 0$. This program was carried out in [3], so far as the spectral properties are concerned, for a large class of interactions. When the imaginary parameter $\varphi$ is "turned on", the essential spectrum rotates to the angle $-2 \varphi$ and splits up into a system of 
"cuts", one starting at each threshold of the system. The continuum eigenvalues become isolated, and new ones ("resonances") may appear between the cuts.

This structure of the spectrum is strongly suggestive of the possibility of decomposing $H(\varphi)$ into a direct sum of operators, each of which has only cut or one point as its spectrum. While the conjecture is immediate, the proof is rather technical. The problem to be solved is that of obtaining estimates of the resolvent between cuts, and the core of this problem is to prove that the connected part of the resolvent goes uniformly to 0 at $\infty$ aliong lines between the cuts.

This problem was solved by van Winter [13] in the case, where the interactions belong to some subclass of the Schmidt class. It was proved in [13] that the Schmidt norm of the connected part of the resolvent goes to 0 at $\infty$ along lines between the cuts, and the consequent decomposition of $H(\varphi)$ was established.

The present paper deals with local potentials, comprising functions which are analytic in an angle, go to 0 as $r^{-\varepsilon}$ at $\infty$ and go at most as $r^{-1+\varepsilon}$ at 0 .

We prove that the norm of the square of the connected part of the resolvent goes to 0 as some negative power of the distance along lines between the cuts (Lemma 5.4). The derived estimates of the resolvent between cuts and the decomposition of $H(\varphi)$ are formulated in Theorems 6.1 and 6.2.

Section 2 contains some basic results which are crucial in obtaining the required estimates. These include certain commutation estimates based on a priori weighted estimates of Agmon [1] and some consequences of the fact, due to Ginibre (private communication) and Agmon [1] that a suitably weighted free resolvent goes to 0 in norm at $\infty$ as the negative square root of the distance along lines parallel to the spectrum.

In Section 3 we derive the estimates for the 2-body problem which serve as the first step in an induction proof on the number $n$ of particles.

In Section 4 we make the induction assumption that all resolvents of Hamiltonians of systems of at most $n-1$ particles have the desired properties and summarize the corresponding properties of the disconnected Hamiltonians of the $n$-body system. These are proved by van Winter $([12,13])$ for interactions in Schmidt class and extended in [14] to local potentials.

The decomposition result again gives rise to immediate conjectures. First of all, it is to be expected that $H$ itself can be decomposed on invariant subspaces $\mathscr{H}_{\mu}^{ \pm}$associated with the thresholds $\mu$, such that the corresponding projections $P_{\mu}^{ \pm}$are the limits of $P_{\mu}\left(e^{i \phi}\right)$ as $\phi \rightarrow 0_{ \pm}$.

Secondly, one would expect under certain conditions on the potential that $\mathscr{H}_{\mu}^{ \pm}$be the range of the channel wave operators $\Omega_{\mu}^{ \pm}$and $\mathscr{H}_{\mu}(\varphi)$ be the range of channel wave operators $\Omega_{\mu}^{+}(\varphi)$ for $\varphi>0$ and $\Omega_{\mu}^{-}(\varphi)$ for $\varphi<0$, such that $\Omega_{\mu}^{+}=$ $\lim _{\varphi \rightarrow 0_{+}} \Omega_{\mu}^{+}(\varphi)$ and $\Omega_{\mu}^{-}=\lim _{\varphi \rightarrow 0} \Omega_{\mu}^{-}(\varphi)$. [It is clear from the structure of $\sigma(H(\varphi))$ that we can only expect to get semigroups corresponding to $t>0$ for $\varphi>0$ and to $t<0$ for $\varphi<0$.] The proof of these two conjectures would then imply asymptotic completeness.

This is in brief outline the program for an analytic scattering theory of manybody system. Although it will involve many technical difficulties, the decomposition result would seem to make it possible to develop an analytic multichannel scattering theory for a large class of potentials. 


\section{Definitions and Notations}

We consider a system $S=\{1, \ldots, n\}$ of $n$ particles, with masses $m_{1}, \ldots, m_{n}$. A cluster $C_{l}$ is a subset $\left\{i_{1}, \ldots, i_{l}\right)$ of $S, i_{1}<\ldots<i_{l}, 2 \leqq l \leqq n$. Let $C_{p}$ be the cluster $\{1, \ldots, p\}$. Let $\bar{r}_{C_{j-1}, j}$ be the position vector from the center of mass of $C_{j-1}$ to particle $j$, and $r_{C_{j-1}, j}=\left|\bar{r}_{C_{j-1}, j}\right|$. We set

$$
\bar{r}_{C_{p}}=\left(\bar{r}_{12}, \bar{r}_{C_{2}, 3}, \ldots, \bar{r}_{C_{p-1}, p}\right)
$$

and

$$
r_{C_{p}}^{2}=r_{12}^{2}+r_{C_{2}, 3}^{2}+\ldots+r_{C_{p-1}, p}^{2} .
$$

Moreover

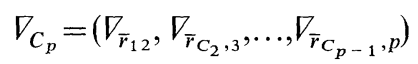

and

$$
\Delta_{C_{p}}=\Delta_{\bar{r}_{12}}+\Delta_{\bar{r}_{C_{2}}, 3}+\ldots+\Delta_{\bar{r}_{C_{p-1}}, p} .
$$

A decomposition $D_{k}$ is a set of disjoint clusters $C^{1}, \ldots, C^{m}$ and single particles $m+1, \ldots, k$. We write $D_{l} \subset D_{k}$ if $l>k$ and every cluster of $D_{l}$ is contained in some cluster of $D_{k}$. We set

$$
\begin{aligned}
\bar{r}_{D_{k}} & =\left(\bar{r}_{C^{1}}, \ldots, \bar{r}_{C^{m}}\right), \\
r_{D_{k}}^{2} & =r_{C^{1}}^{2}+\ldots+r_{C^{m}}^{2}, \\
\nabla_{D_{k}} & =\left(\nabla_{C^{1}}, \ldots, \nabla_{C^{m}}\right), \\
\Delta_{D_{k}} & =\Delta_{C^{1}}+\ldots+\Delta_{C^{m}} .
\end{aligned}
$$

We denote by $H_{0, D_{k}}^{\mathrm{rel}}$ the free Hamiltonian of the centers of mass of the clusters $C^{1}, \ldots, C^{m}$ and the single particles in $S \backslash\left\{C^{1} \cup \ldots \cup C^{m}\right\}$. Let $l_{i}$ be the number of particles in the cluster $C^{i}$, let $p=\left(l_{1}-1\right)+\ldots+\left(l_{m}-1\right)$ be the total number of internal position vectors of the clusters $C^{1}, \ldots, C^{m}$, and set

$$
\begin{aligned}
& \left(\bar{r}_{1}, \ldots, \bar{r}_{p}\right)=\left(\bar{r}_{C^{1}}, \ldots, \bar{r}_{C^{m}}\right), \\
& \bar{r}_{i}=\left(x_{i 1}, x_{i 2}, x_{i 3}\right), \Delta_{i}=\frac{\partial^{2}}{\partial x_{i 1}^{2}}+\frac{\partial^{2}}{\partial x_{i 2}^{2}}+\frac{\partial^{2}}{\partial x_{i 3}^{2}}, \quad i=1, \ldots, p .
\end{aligned}
$$

With this choice of coordinates, the free Hamiltonian of the system $S$ in the center of mass frame of reference is of the form

$$
H_{0}=-\mu_{1} \Delta_{1}-\mu_{2} \Delta_{2}-\ldots-\mu_{p} \Delta_{p}+H_{0, D}^{\mathrm{rel}}
$$

where the $\mu_{i}>0$ are certain functions of $m_{1}, \ldots, m_{n}$.

We shall need the following estimates.

Lemma 1.1. (1) If $D_{l} \subset D_{k}$, then

$$
1+r_{D_{l}}^{2} \leqq C\left(1+r_{D_{k}}^{2}\right) \text {. }
$$

(2) If the particle $j$ does not belong to any of the clusters of $D_{k+1}$, if $D_{k}$ is obtained by adding $j$ to one of the clusters $C$ of $D_{k+1}$, and $i \in C$, then

$$
1+r_{D_{k}}^{2} \leqq\left(1+r_{D_{k+1}}^{2}\right)\left(1+r_{i j}^{2}\right) \text {. }
$$


(3) If $C^{1}$ and $C^{2}$ are clusters of the decomposition $D_{k+1}$, if $i \in C^{1}$ and $j \in C^{2}$, and $D_{k}$ is obtained from $D_{k+1}$ by joining $C^{1}$ and $C^{2}$, then

$$
1+r_{D_{k}}^{2} \leqq\left(1+r_{D_{k+1}}^{2}\right)\left(1+r_{i j}^{2}\right) \text {. }
$$

Proof. It is clear that (1) holds, and that (2) and (3) are reduced to proving

(4) $1+r_{C \cup j}^{2} \leqq\left(1+r_{C}^{2}\right)\left(1+r_{i j}^{2}\right)$

and

(5) $1+r_{C^{1} \cup C^{2}}^{2} \leqq\left(1+r_{D}^{2}\right)\left(1+r_{i j}^{2}\right)$,

where $D$ is the decomposition containing only the clusters $C^{1}$ and $C^{2}$. The inequalities (4) and (5) are easily proved by induction using the triangle inequality and the fact that the distance from the particle $j$ to the center of mass of the cluster $C$ is proportional to the distance from $j$ to the center of mass of $C \cup j$.

In all our estimates, $C$ denotes different positive constants, also possibly on two sides of an inequality.

\section{Some Basic Estimates}

In this section we prove some results which are of basic importance for the estimate of the connected part of the resolvent. First we derive some commutation estimates on the basis of the following result of Agmon [1].

Lemma 2.1. Let $\alpha>\frac{1}{2}$ be a fixed constant. Let the coordinate vectors $\bar{r}_{1}, \ldots, \bar{r}_{n-1}$ be chosen as in Section 1, such that $H_{0}$ is of the form

$$
H_{0}=-\mu_{1} \Delta_{1}-\mu_{2} \Delta_{2}-\ldots-\mu_{n-1} \Delta_{n-1} \text {. }
$$

There exists a constant $C$ independent of $\lambda \in \mathbb{C}$ such that for all $f \in \mathscr{D}_{H_{0}}$ and $i=1, \ldots, n-1, j=1,2,3$, we have

$$
\left\|\left(1+x_{i j}^{2}\right)^{-(\alpha / 2)} \frac{\partial f}{\partial x_{i j}}\right\|^{2} \leqq C\left\|\left(1+x_{i j}^{2}\right)^{(\alpha / 2)}\left(H_{0}-\lambda\right) f\right\|^{2} .
$$

This is a special case of [1], Lemma A2.

Let $D$ be a decomposition and let $\bar{r}_{1} \ldots \bar{r}_{p}$ be coordinate vectors chosen relative to $D$ as in Section 1 . Then $x_{i j}^{2} \leqq r_{D}^{2}$ for $i=1 \ldots p, j=1,2,3$, and hence

$$
\left\|\left(1+r_{D}^{2}\right)^{-(\alpha / 2)} \frac{\partial f}{\partial x_{i j}}\right\|^{2} \leqq C\left\|\left(1+r_{D}^{2}\right)^{(\alpha / 2)}\left(H_{0}-\lambda\right) f\right\|^{2} .
$$

Summing (2) over $i$ and $j$ we obtain for $\lambda \in \mathbb{C}, f \in \mathscr{D}_{H_{0}}$

$$
\left\|\left(1+r_{D}^{2}\right)^{-(\alpha / 2)} \nabla_{D} f\right\|^{2} \leqq C\left\|\left(1+r_{D}^{2}\right)^{(\alpha / 2)}\left(H_{0}-\lambda\right) f\right\|^{2} \text {. }
$$

From (3) we derive the following estimate.

Lemma 2.2. Let $\alpha>\frac{1}{2}, \beta<\frac{1}{2}$ be fixed constants, and let $D$ be a decomposition. For $|\operatorname{Im} \lambda| \geqq \varepsilon>0$ and for all $f \in \mathscr{H}$ we have

$$
\left\|\left(1+r_{D}^{2}\right)^{-(\alpha / 2)} R_{0}(\lambda) f\right\| \leqq C_{\varepsilon}\left\|R_{0}(\lambda)\left(1+r_{D}^{2}\right)^{-(\beta / 2)} f\right\| .
$$


Proof. The inequality (4) for all $f \in \mathscr{H}$ is equivalent to

$$
\left\|\left(1+r_{D}^{2}\right)^{-(\alpha / 2)} R_{0}(\lambda)\left(1+r_{D}^{2}\right)^{(\beta / 2)}\left(H_{0}-\lambda\right)\right\| \leqq C_{\varepsilon} \text { for }|\operatorname{Im} \lambda| \geqq \varepsilon
$$

or, taking adjoints, equivalent to

$$
\left\|\left(H_{0}-\lambda\right)\left(1+r_{D}^{2}\right)^{(\beta / 2)} R_{0}(\lambda)\left(1+r_{D}^{2}\right)^{-(\alpha / 2)}\right\| \leqq C_{\varepsilon} \text { for }|\operatorname{Im} \lambda| \geqq \varepsilon .
$$

To simplify notation, we assume that all the coefficients $\mu_{i}, i=1 \ldots k$, are equal to 1 , so that

$$
H_{0}=-\Delta_{D}+H_{0}^{\mathrm{rel}} \text {. }
$$

Then the operator, whose norm has to be estimated by (6), is

$$
\begin{gathered}
\left(H_{0}-\lambda\right)\left(1+r_{D}^{2}\right)^{(\beta / 2)} R_{0}(\lambda)\left(1+r_{D}^{2}\right)^{-(\alpha / 2)} \\
=\left(1+r_{D}^{2}\right)^{((\beta-\alpha) / 2)} I-2\left(\left[\nabla_{D}\left(1+r_{D}^{2}\right)^{(\beta / 2)}\right] \cdot \nabla_{D}\right) R_{0}(\lambda)\left(1+r_{D}^{2}\right)^{-(\alpha / 2)} \\
-\left[\Delta_{D}\left(1+r_{D}^{2}\right)^{(\beta / 2)}\right] R_{0}(\lambda)\left(1+r_{D}^{2}\right)^{-(\alpha / 2)} \\
=\left(1+r_{D}^{2}\right)^{(\beta-\alpha) / 2} I-2 \beta\left(1+r_{D}^{2}\right)^{(\beta / 2)-1}\left(r_{D} \cdot \nabla_{D}\right) R_{0}(\lambda)\left(1+r_{D}^{2}\right)^{-(\alpha / 2)} \\
+\left[\beta k\left(1+r_{D}^{2}\right)^{(\beta / 2)-1}+(\beta / 2)((\beta / 2)-1) r_{D}^{2}\left(1+r_{D}^{2}\right)^{(\beta / 2)-2}\right] \\
R_{0}(\lambda)\left(1+r_{D}^{2}\right)^{-(\alpha / 2)} .
\end{gathered}
$$

On the right-hand side of (7), the first term is a bounded operator, and the last term is bounded by $C\left\|R_{0}(\lambda)\right\|$. In order to obtain (6) it therefore suffices to show

$$
\left\|\left(1+r_{D}^{2}\right)^{(\beta / 2)-1} \nabla_{D} f\right\|^{2} \leqq C_{\varepsilon}\left\|\left(1+r_{D}^{2}\right)^{\alpha / 2}\left(H_{0}-\lambda\right) f\right\|^{2}
$$

for all $f \in \mathscr{D}_{\left(1+r_{D}^{2}\right)^{\alpha / 2}\left(H_{0}-\lambda\right)}$ and $|\operatorname{Im} \lambda| \geqq \varepsilon>0$.

This follows from (3), since $\beta<\frac{1}{2}, \alpha>\frac{1}{2}$, in fact with $C_{\varepsilon}$ independent of $\varepsilon$, and (4) is proved.

We shall also make use of the following result due to Ginibre (private communication), cf. also [5], and Agmon ([1], Appendix A, Remark 2).

Lemma 2.3. Let $\alpha>\frac{1}{2}$ be fixed. Then for $\lambda=s+i t \in \mathbb{C}$

$$
\left\|\left(1+r_{S}^{2}\right)^{-(\alpha / 2)} R_{0}(\lambda)\left(1+r_{S}^{2}\right)^{-(\alpha / 2)}\right\|<C s^{-1 / 2} \quad \text { for } \quad s>K .
$$

A simple, but useful observation is the following.

Lemma 2.4. Let $M$ be a subset of $\mathbb{C}$, and let $A(\lambda)$ be a function from $M$ into $\mathscr{B}(\mathscr{H})$, such that

$$
\|A(\lambda)\| \leqq C \quad \text { for } \quad \lambda \in M .
$$

Then for any decomposition $D$

$$
\left\|\left(1+r_{D}^{2}\right)^{-\varepsilon} A(\lambda)\right\| \leqq C\left\|\left(1+r_{D}^{2}\right)^{-2^{p} \varepsilon} A(\lambda)\right\|^{2-p} \quad \text { for } \quad \lambda \in M .
$$

Proof. We have

$$
\left\|\left(1+r_{D}^{2}\right)^{-\varepsilon} A(\lambda)\right\|=\left\|A^{*}(\lambda)\left(1+r_{D}^{2}\right)^{-2 \varepsilon} A(\lambda)\right\|^{1 / 2} \leqq C\left\|\left(1+r_{D}^{2}\right)^{-2 \varepsilon} A(\lambda)\right\|^{1 / 2} .
$$


Repeating this argument, we obtain the Lemma.

From Lemma 2.3 follows

Lemma 2.5. Let $\alpha>\frac{1}{2}$ be fixed. Then

$$
\left\|\left(1+r_{S}^{2}\right)^{-(\alpha / 2)} R_{0}(s+i t)\right\| \leqq C_{\varepsilon} s^{-(1 / 4)} \quad \text { for } \quad|t| \geqq \varepsilon>0, s>K .
$$

Proof. By the first resolvent equations and Lemma 2.3,

$$
\begin{aligned}
& \left\|\left(1+r_{S}^{2}\right)^{-(\alpha / 2)} R_{0}(s+i t)\right\|^{2} \\
& \quad=(2 t)^{-1 / 2}\left\|\left(1+r_{S}^{2}\right)^{-(\alpha / 2)}\left[R_{0}(s+i t)-R_{0}(s-i t)\right]\left(1+r_{S}^{2}\right)^{-(\alpha / 2)}\right\|^{1 / 2} \\
& \quad \leqq C \varepsilon^{-1 / 2} S^{-(1 / 4)} .
\end{aligned}
$$

We shall make use of the following estimates due to Iorio and O'Carroll [6].

Lemma 2.6. Let $w_{i j}=u_{i j}+q_{i j}$, where $u_{i j} \in L^{p}\left(R^{3}\right)$ for some $p>3$ and $q_{i j} \in L^{\infty}\left(R^{3}\right)$, $i<j=1, \ldots, n$.

Then for all $i, j, k, l$ and $|\operatorname{Im} \lambda| \geqq \varepsilon>0$ we have

(i) $\left\|w_{i j} R_{0}(\lambda) w_{k l}\right\| \leqq C_{\varepsilon}$

and

(ii) $\left\|w_{i j} R_{0}(\lambda)\right\| \leqq C_{\varepsilon}$.

Proof. Since $q_{i j} \in L^{\infty}\left(R^{3}\right)$, it suffices to prove that for $|\operatorname{Im} \lambda| \geqq \varepsilon>0$ we have

$$
\left\|u_{i j} R_{0}(\lambda) u_{k 1}\right\| \leqq C_{\varepsilon}
$$

and

$$
\left\|u_{i j} R_{0}(\lambda)\right\| \leqq C_{\varepsilon} .
$$

The inequalities (11) are proved in (6), and (12) follows from (11), using

$$
\left\|u_{i j} R_{0}(\lambda)\right\|=\left(\frac{1}{2|\operatorname{Im} \lambda|}\left\|u_{i j}\left[R_{0}(\lambda)-R_{0}(\bar{\lambda})\right] u_{i j}\right\|\right)^{1 / 2} .
$$

Lemma 2.7. Let I be a closed interval and let

$$
S_{I}=\{\lambda=s+i t \mid s \in R, t \in I\} .
$$

Suppose that $B(\lambda)$ is a continuous function from $S_{I}$ into $\mathscr{B}(\mathscr{H})$, which is analytic in the interior $\stackrel{\circ}{S}_{I}$ of $S_{I}$ and satisfies

$$
\int_{-\infty}^{\infty}\|B(s+i t) f\|^{2} d t \leqq C_{I}\|f\|^{2} \quad \text { for } \quad t \in I, f \in \mathscr{H} .
$$

Then

$$
\|B(\lambda)\| \leqq C \quad \text { for } \quad \lambda \in S_{I} .
$$

Proof. For $f, g \in \mathscr{H}$ the function $(f, B(\lambda) g)$ is analytic in $\stackrel{S}{I}_{I}$ and

$$
\int_{-\infty}^{\infty}|(f, B(s+i t) g)|^{2} d s<C \quad \text { for } \quad t \in I
$$


This implies (cf. [8]) that

$$
|(f, B(s+i t) g)| \leqq C \text { for } s \in R, t \in I .
$$

Then the Lemma follows from the uniform boundedness principle.

\section{The 2-Body Problem}

We consider the Schrödinger operator in $\mathscr{H}=L^{2}\left(R^{3}\right)$ given by

$$
H=H_{0}+v \text {, }
$$

where $H_{0}$ is the self-adjoint operator associated with $-\Delta$, and $v$ is the maximal multiplication operator corresponding to a real-valued function $v(\vec{r})$ on $R^{3}$ satisfying the following conditions.

(i) $v=(1+r)^{-\varepsilon}(u+w)$,

where $u$ and $w$ can best be described in polar coordinates.

Let $0<a<\frac{\pi}{2}$, let $S_{a}$ be the angular sector

$$
S_{a}=\left\{z=\varrho e^{i \varphi} \mid 0<\varrho<\infty,-a<\varphi<a\right\},
$$

and let $\Omega$ be the unit sphere in $R^{3}$.

The functions $u$ and $w$ are restrictions to $(0, \infty)$ of analytic functions $u(z)$ and $w(z)$ from $S_{a}$ into $L^{2}(\Omega)$ satisfying the following conditions for some $p>\frac{3}{2}$.

$$
\begin{aligned}
& \text { (ii) } \sup _{|\varphi| \leqq a-\varepsilon}\left\{\int_{1}^{\infty}\left\|u\left(r e^{i \varphi}\right)\right\|_{L^{p}(\Omega)}^{p} r^{2} d r\right\}<C_{\varepsilon} . \\
& \text { (iii) } \sup _{|\varphi| \leqq a-\varepsilon}\left\{\int_{0}^{\infty}\left\|u\left(r e^{i \varphi}\right)\right\|_{L^{2} p_{(\Omega)}}^{2 r^{2}} d r\right\}<C_{\varepsilon} . \\
& \text { (iv) } \sup _{|\varphi| \leqq a-\varepsilon}\left\{\sup _{0<r<\infty}\left\|q\left(r e^{i \varphi}\right)\right\|_{L^{\infty}(\Omega)}\right\}<C_{\varepsilon} .
\end{aligned}
$$

For radial potentials $u(r)$ and $w(r)$ these conditions reduce to the requirement that $u$ and $w$ be restrictions to $R^{+}$of analytic functions on $S_{a}$ satisfying (ii)-(iv) with

$$
\left\|u\left(r e^{i \varphi}\right)\right\|_{L^{p}(\Omega)} \text { and }\left\|u\left(r e^{i \varphi}\right)\right\|_{L^{2 p}(\Omega)}
$$

replaced by $\left|u\left(r e^{i \varphi}\right)\right|$ and $\left\|q\left(r e^{i \varphi}\right)\right\|_{L^{\infty}(\Omega)}$ by $\left|q\left(r e^{i \varphi}\right)\right|$.

The condition on the potential is satisfied by a radial function $v(r)$, which is the restriction to $(0, \infty)$ of a function $v(z)$ analytic in the angle $S_{a}$ and such that

$$
\begin{gathered}
\left|v\left(r e^{i \varphi}\right)\right|=0\left(r^{-1+\varepsilon}\right) \text { for } \quad r \rightarrow 0, \\
|v(r)|=0\left(r^{-\varepsilon}\right) \text { for } \quad r \rightarrow \infty
\end{gathered}
$$

uniformly in every angle $\{|\varphi| \leqq a-\delta\}$.

Conditions (ii) and (iii) imply

$$
\sup _{|\varphi| \leqq a-\varepsilon}\left\{\int_{0}^{1}\left\|u\left(r e^{i \varphi}\right)\right\|_{L^{2}(\Omega)}^{2} r^{2} d r\right\}<C_{\varepsilon}
$$


and

(vi) $\sup _{|\varphi| \leqq a-\varepsilon} \sup _{1 \leqq R<\infty}\left\{\int_{R}^{R+1}\left\|u\left(r e^{i \varphi}\right)\right\|_{L^{2}(\Omega)}^{2} r^{2} d r\right\}<C_{\varepsilon}$.

It follows that $v$ satisfies (v), (vi) and

(vii) $\sup _{|\varphi| \leqq a-\varepsilon}\left\{\int_{R}^{R+1}\left\|v\left(r e^{i \varphi}\right)\right\|_{L^{2}(\Omega)}^{2} r^{2} d r\right\} \underset{R \rightarrow \infty}{\longrightarrow} 0$.

It then follows from [2] Theorem 4.1, that $v$ is $S_{a}$-dilation-analytic and $H_{0^{-}}$ compact. We recall the meaning of this. Let $U(\varrho)$ be the dilation group on $\mathscr{H}$ defined by

$$
(U(\varrho) f) \bar{r})=\varrho^{3 / 2} f(\bar{r})
$$

and let

$$
v(\varrho)=U(\varrho) v U(\varrho)^{-1} .
$$

Then $v(\varrho)$ is restriction to $(0, \infty)$ of an analytic function $v(z)$ from $S_{a}$ into the space of compact operators from $\mathscr{D}_{\mathrm{H}_{0}}$ into $\mathscr{H}$.

We consider the self-adjoint analytic family of type $A$ in the sense of Kato defined for $z \in S_{a}$ by

$$
H(z)=z^{-2} H_{0}+v(z) \text {. }
$$

The spectrum of $H(z)=H\left(\varrho e^{i \varphi}\right)$ (cf. [3]) depends only on $\varphi$ and consists of

(1) the essential spectrum $\sigma_{e}(\varphi)=e^{-2 i \varphi} R^{+}$

(2) the $\varphi$-independent discrete spectrum $\sigma_{d}(\varphi)$ contained in the angle $\left(R^{+}\right.$, $e^{-2 i \varphi} R^{+}$) and with 0 as the only possible accumulation point.

In what follows, we keep $z=\varrho e^{i \varphi}$ fixed with $\varphi \neq 0$, letting for simplicity $\varrho=1$ and set $H(\varphi)=H\left(e^{i \varphi}\right), R(\lambda, \varphi)=R\left(\lambda, e^{i \varphi}\right)$ etc.

We shall now establish the properties of the operator $H(\varphi)$ which will be used in the following sections, and which provide the first step in the induction proof of the same properties for the $n$-body Hamiltonian.

Let $I$ be a closed interval not containing 0 , such that the strip

$$
S_{I}(\varphi)=\left\{\lambda=e^{-2 i \varphi}(s+i t) \mid s \in R, t \in I\right\}
$$

is contained in the resolvent set $\varrho(H(\varphi))$.

Lemma 3.1. There exists $\delta>0$ such that

$$
\left\|R_{0}\left(e^{-2 i \varphi}(s+i t), \varphi\right) v(\varphi) R_{0}\left(e^{-2 i \varphi}(s+i t), \varphi\right) v(\varphi)\right\| \leqq C s^{-\delta} \quad \text { for } \quad s \in R, t \in I .
$$

Proof. By Lemma 2.6, we have

$$
\begin{aligned}
& \left\|R_{0}\left(e^{-2 i \varphi}(s+i t), \varphi\right) v(\varphi) R_{0}\left(e^{-2 i \varphi}(s+i t), \varphi\right) v(\varphi)\right\| \\
& \leqq C_{I}\left\|R_{0}\left(e^{-2 i \varphi}(s+i t), \varphi\right)\left(1+e^{i \varphi} r\right)^{-\varepsilon}\right\| \cdot\left\|w(\varphi) R_{0}\left(e^{-2 i \varphi}(s+i t), \varphi\right) w(\varphi)\right\| \\
& \leqq C_{I}\left\|R_{0}(s+i t)(1+r)^{-\varepsilon}\right\| \text { for } \quad s \in R, t \in I .
\end{aligned}
$$

The function $\left\|R_{0}(s+i t)(1+r)^{-\varepsilon}\right\|$ is bounded by $C s^{-\delta}$ for $s \in R, t \in I$ with some $\delta>0$ by Lemmas 2.4 and 2.5 (ii), and the Lemma is proved. 
Lemma 3.2. For $t \in I, f \in \mathscr{H}$ we have

$$
\int_{-\infty}^{\infty}\left\|R\left(e^{-2 i \varphi}(s+i t), \varphi\right) f\right\|^{2} d t \leqq C_{I}\|f\|^{2} .
$$

Proof. By iteration of the second resolvent equation we obtain for $\lambda \in \varrho(H(\varphi))$

$$
R(\lambda, \varphi)=R_{0}(\lambda, \varphi)-R_{0}(\lambda, \varphi) v(\varphi) R_{0}(\lambda, \varphi)+R_{0}(\lambda, \varphi) v(\varphi) R_{0}(\lambda, \varphi) v(\varphi) R(\lambda, \varphi) .
$$

For $\lambda=e^{-2 i \varphi}(s+i t), s \in R, t \in I$, this yields

$$
\begin{aligned}
R\left(e^{-2 i \varphi}(s+i t), \varphi\right)= & \left(1-R_{0}\left(e^{-2 i \varphi}(s+i t), \varphi\right) v(\varphi) R_{0}\left(e^{-2 i \varphi}(s+i t), \varphi\right) v(\varphi)\right)^{-1} \\
& \left(1-R_{0}\left(e^{-2 i \varphi}(s+i t), \varphi\right) v(\varphi)\right) R_{0}\left(e^{-2 i \varphi}(s+i t), \varphi\right) .
\end{aligned}
$$

By Lemma 3.1, the norm of the first factor is bounded for $t \in I, s>K$. It is clearly bounded for large negative $s$ and hence by continuity bounded for all $s \in R, t \in I$. The norm of the second factor is bounded for $s \in R, t \in I$ by Lemma 2.6. Hence $\left\|R\left(e^{-2 i \varphi}(s+i t), \varphi\right) f\right\| \leqq C_{I}\left\|R_{0}\left(e^{-2 i \varphi}(s+i t), \varphi\right) f\right\|=C_{I}\left\|R_{0}(s+i t) f\right\|$ for $s \in R$, $t \in I$, and the Lemma follows.

Corollary 3.3. $\|R(\lambda, \varphi)\| \leqq C$ for $\lambda \in S_{I}(\varphi)$.

Proof. This follows from Lemma 2.7 and Lemma 3.2 and also directly from the above expression for $R(\lambda, \varphi)$.

\section{The $n$-Body Problem}

We shall now consider a system $S$ of $n$ particles $1, \ldots, n$ interacting through 2 -body potentials $v_{i j}\left(\bar{r}_{i j}\right), i<j=, \ldots, n$.

The real-valued functions $v_{i j}\left(r_{i j}\right)$ on $R^{3}$ are assumed to satisfy conditions (i)-(iv) of Section 3. This implies that the multiplication operators $v_{i j}$ corresponding to $v_{i j}\left(\bar{r}_{i j}\right)$ are $H_{0}-\varepsilon$-bounded, and hence a unique self-adjoint operator $H$ is defined on $\mathscr{D}_{H}=H_{2}\left(R^{3(n-1)}\right)$ by

$$
H=H_{0}+\sum_{i<j=1} v_{i j} \text {. }
$$

As in the 2-body problem we introduce the self-adjoint analytic family $H(z)$ defined for $z \in S_{a}$ by

$$
H(z)=z^{-2} H_{0}+\sum_{i<j=1}^{n} v_{i j}(z) .
$$

For a cluster $C$ we set $\mathscr{H}^{C}=L^{2}\left(\bar{r}_{C}\right)$ and $H^{C}(z)$ denotes the operator in $\mathscr{H}^{C}$ defined by

$$
H^{C}(z)=z^{-2} H_{0}^{C}(z)+\sum_{i<j=1}^{n} v_{i j}(z)
$$

We identify $H^{C}(z)$ with the operator $H^{C}(z) \otimes I_{\text {rel }}^{C}$ in $\mathscr{H}$, where $I_{\text {rel }}^{C}$ is the identity on $L^{2}\left(r_{C}^{\mathrm{rel}}\right)$ and $\bar{r}_{C}^{\mathrm{rel}}$ denotes internal coordinate vectors of the center of mass of $C$ and the particles in $S \backslash C$.

If $C_{1}, \ldots, C_{m}$ are disjoint clusters, we set

$$
H^{C_{1}, \ldots, C_{m}}(z)=H^{C_{1}}(z)+H^{C_{2}}(z)+\ldots+H^{C_{m}}(z) \text {. }
$$


If $D_{k}$ is the decomposition with clusters $C_{1}, \ldots, C_{m}$, we set

$$
H_{D_{k}}(z)=H^{C_{1}, \ldots, C_{m}}(z)+H_{0, \text { rel }}^{D_{k}}(z)=H_{0}+\sum_{l=1}^{m} \sum_{i j \in C_{l}} v_{i j}(z)
$$

where $H_{0 \text {,rel }}^{D_{k}}(z)$ is the free Hamiltonian of the centers of mass of $C_{1}, \ldots, C_{m}$ and the particles of $S \backslash\left\{C_{1} \cup \ldots \cup C_{m}\right\}$.

We shall also denote pairs $(i j)$ by $\alpha$. We shall for simplicity of notation take $z=e^{i \varphi}, \varphi \neq 0$, and set

$$
H(z)=H(\varphi), R(\lambda, z)=R(\lambda, \varphi), R^{C}(\lambda, z)=\left(H^{C}(\varphi)-\lambda\right)^{-1} \quad \text { etc. }
$$

The basic tool in the study of $R(\lambda, \varphi)$ is the Weinberg-van Winter equation, which we formulate in two forms as follows.

The $l$-connected and the $r$-connected parts $I_{D_{k}}^{l}(\lambda, \varphi)$ and $I_{D_{k}}^{r}(\lambda, \varphi)$ of $R_{D_{k}}(\lambda, \varphi)$ are defined by

$$
\begin{array}{r}
I_{D_{k}}^{l}(\lambda, \varphi)=\sum_{D_{n-1} \subset \ldots \subset D_{k}\left(\alpha_{1} \ldots \alpha_{n-k}\right)} R_{0}(\lambda, \varphi) v_{\alpha_{1}}(\varphi) R_{D_{n-1}}(\lambda, \varphi) \\
v_{\alpha_{2}}(\varphi) \ldots R_{D_{k+1}}(\lambda, \varphi) v_{\alpha_{n-k}}(\varphi)
\end{array}
$$

and

$$
\begin{aligned}
I_{D_{k}}^{r}(\lambda, \varphi)=\sum_{D_{n-1} \subset \ldots \subset D_{k}\left(\alpha_{1} \ldots \alpha_{n-k}\right)} \sum_{\alpha_{n-k}}(\varphi) R_{D_{k+1}}(\lambda, \varphi) \ldots \\
v_{\alpha_{2}}(\varphi) R_{D_{n-1}}(\lambda, \varphi) v_{\alpha_{1}}(\varphi) R_{0}(\lambda, \varphi),
\end{aligned}
$$

where

$$
\sum_{D_{n-1} \subset \ldots \subset D_{k}}
$$

is over all chains of decompositions $D_{n-1} \subset \ldots \subset D_{k+1}$ such that $D_{j}$ arises from $D_{j+1}$ by joining two clusters of $D_{j+1}$, and

$$
\sum_{\left(\alpha_{1} \ldots \alpha_{n-k}\right)}
$$

is over all $\left(\alpha_{1} \ldots \alpha_{n-k}\right)$ such that the particles of the pair $\alpha_{n-j}$ belong to the same cluster of $D_{j}$ but are single or belong to different clusters of $D_{j+1}$, in other words $\alpha_{n-j}$ connects clusters of $D_{j+1}$ to obtain $D_{j}, j=k, \ldots, n-1$.

We then have the following two forms of the Weinberg-van Winter equation for $R_{D_{k}}(\lambda, \varphi)$, valid for $\lambda \in \varrho\left(H_{D_{k}}(\varphi)\right)$.

$$
\begin{array}{ll}
\text { l-form } & R_{D_{k}}(\lambda, \varphi)=\sum_{D_{m} \subset D_{k}}(-1)^{m-1}(m-1) ! R_{D_{m}}(\lambda, \varphi)+I_{D_{k}}^{l}(\lambda, \varphi) R_{D_{k}}(\lambda, \varphi) \\
\text { r-form } & R_{D_{k}}(\lambda, \varphi)=\sum_{D_{m} \subset D_{k}}(-1)^{m-1}(m-1) ! R_{D_{m}}(\lambda, \varphi)+R_{D_{K}}(\lambda, \varphi) I_{D_{k}}^{R}(\lambda, \varphi) .
\end{array}
$$

The $l$-form is the one given in [12 II (6.38)]. The $r$-form is obtained in the same way from the Born series

$$
R_{D_{k}}(\lambda, \varphi)=\sum_{m=0}^{\infty} \sum_{\left(\alpha_{1} \ldots \alpha_{m}\right)} R_{0}(\lambda, \varphi) v_{\alpha_{1}}(\varphi) R_{0}(\lambda, \varphi) v_{\alpha_{2}}(\varphi) \ldots v_{\alpha_{m}}(\varphi) R_{0}(\lambda, \varphi)
$$


where the $\left(\alpha_{1} \ldots \alpha_{m}\right)$ vary over all sets of pairs which connect particles in some cluster of $D_{k}$.

For $k=1$ we obtain the equations for $R(\lambda, \varphi)$.

We set

$$
I_{D_{1}}^{l}(\lambda, \varphi)=I^{l}(\lambda, \varphi), I_{D_{1}}^{r}(\lambda, \varphi)=I^{r}(\lambda, \varphi) .
$$

Our basic objective is to estimate $R(\lambda, \varphi)$ on lines $\left\{\lambda=e^{-2 i \varphi}(s+i t) \mid s \in R\right\}$ between cuts, and the method for obtaining this is by proving that

$$
\left\|\left(I^{l}\left(e^{-2 i \varphi}(s+i t, \varphi)\right)\right)^{2}\right\| \underset{s \rightarrow \infty}{\longrightarrow} 0 .
$$

and solving the iterated Equation (1) for $R(\lambda, \varphi)$.

In order to obtain this we need uniform estimates on the norms of the operators $R_{D}\left(e^{-2 i \varphi}(s+i t), \varphi\right)$ for $s \in R$. Such estimates were obtained by van Winter ([12 II, 13]), using convolution techniques. These results can be extended to our class of potentials by establishing certain additional estimates to justify the different steps of the proofs of [12 II] and [13]. This is carried out in detail in [14]. We quote here the result required for the estimate of $\left\|\left(I^{l}(\lambda, \varphi)\right)^{2}\right\|$.

We make the following induction assumption on the operators $H^{C}(\varphi)$, where $C$ is any cluster of at most $n-1$ particles and $\varphi \neq 0$. We notice that these assumptions are verified in Section 3 in the two-body case. The set of thresholds $\mu_{C}(\varphi)$ of $H^{C}(\varphi)$ is denoted by $\Sigma_{C}(\varphi)$.

\section{Induction Assumption}

(I1) $\sigma_{e}\left(H^{C}(\varphi)\right)=\left\{\mu_{C}(\varphi)+e^{-2 i \varphi} R^{+} \mid \mu_{C}(\varphi) \in \Sigma_{C}(\varphi)\right\}$.

(I2) $\sigma_{d}\left(H^{C}(\varphi)\right)$ is contained in the angular sector formed by the half-lines $\lambda_{e, C}+R^{+}$ and $\lambda_{e, C}+e^{-2 i \varphi} R^{+}$, where $\lambda_{e, C}=\min \left\{\Sigma_{C}(\varphi) \cap R\right\}$. The points in $\sigma_{d}\left(H_{C}(\varphi)\right)$ are independent of $\varphi$ unless absorbed by $\sigma_{e}\left(H_{C}(\varphi)\right)$ and accumulate at most at thresholds or at $\infty$. For any two neighboring cuts and any $\varepsilon>0$ there exists $K(\varepsilon)>0$ such that there are no resonances in the strip between the cuts at distance $\geqq \varepsilon$ from the cuts, provided the distance along the cuts is greater than $K(\varepsilon)$. Similarly, in the half-plane to the right of the cut corresponding to the threshold $\mu$ with largest $\operatorname{Re} \mu$ there are no resonances at distance $\geqq \varepsilon$ from the cut, provided the distance along the cut is greater than $K(\varepsilon)$.

(I3) For any closed interval $I$, such that $S_{I}(\varphi) \subset \varrho\left(H^{c}(\varphi)\right)$, we have

$$
\int_{-\infty}^{\infty}\left\|R^{C}\left(e^{-2 i \varphi}(s+i t), \varphi\right) f\right\|^{2} d s \leqq C_{I}\|f\|^{2} \quad \text { for } \quad t \in I, f \in \mathscr{H} .
$$

We notice that (I3) implies

$$
\int_{-\infty}^{\infty}\left\|R^{C^{*}}\left(e^{-2 i \varphi}(s+i t), \varphi\right) f\right\|^{2} d s \leqq C_{I}\|f\|^{2} \quad \text { for } \quad t \in I, f \in \mathscr{H},
$$

since

$$
R^{C^{*}}\left(e^{-2 i \varphi}(s+i t), \varphi\right)=R^{C}\left(e^{2 i \varphi}(s-i t),-\varphi\right) .
$$


Also, by Lemma 2.7, (I3) and (I4) imply

$$
\begin{aligned}
& \left\|R^{C}(\lambda, \varphi)\right\| \leqq C \text { for } \lambda \in S_{I}(\varphi) \\
& \left\|R^{C^{*}}(\lambda, \varphi)\right\| \neq C \text { for } \lambda \in S_{I}(\varphi) .
\end{aligned}
$$

We notice, as was pointed out by Simon [10], that the assumptions (I1), (I2) on the spectrum of $H^{C}(\varphi)$ imply that $H^{C}(\varphi)$ is sectorial with arbitrarily small opening angle, and hence

$$
\left\|R^{C}(\lambda, \varphi)\right\| \leqq C|\lambda|^{-1}
$$

for $\lambda$ in any fixed angular sector contained in $\varrho\left(H^{C}(\varphi)\right)$, directed away from $e^{-2 i \varphi}$ Definition. We define $\left[R^{C_{1}} * R^{C_{2}}\right](\lambda, \varphi)$ by

$$
\left[R^{C_{1}} * R^{C_{2}}\right](\lambda, \varphi) f=\frac{1}{2 \pi i} \int_{\Gamma_{1}} R^{C_{1}}(\lambda-\mu, \varphi) R^{C_{2}}(\mu, \varphi) f d \mu \text { for } f \in \mathscr{H}
$$

and

$$
\left[R^{C_{1}} * R^{C_{2}} * \ldots * R^{C_{k}} * R_{\mathrm{rel}}^{D}\right](\lambda, \varphi)
$$

inductively in the same way.

On the basis of the induction assumption on the operators $R^{C}(\lambda, \varphi)$ we obtain the following result on the operators $R_{D}(\lambda, \varphi)$.

Lemma 4.1. Let $D$ be the decomposition corresponding to the clusters $C_{1}, \ldots, C_{k}$, and let I be a closed interval such that $S_{I}(\varphi) \subset \bigcap_{i=1}^{k} \varrho\left(H^{C_{i}}(\varphi)\right)$. Then

(i) $R_{D}(\lambda, \varphi)=\left[R^{C_{1}} * R^{C_{2}} * \ldots * R^{C_{k}} * R_{\mathrm{rel}}^{D}\right](\lambda, \varphi)$

(ii) $\int_{-\infty}^{\infty}\left\|R_{D}\left(e^{-2 i \varphi}(s+i t), \varphi\right) f\right\|^{2} d s \leqq C_{I}\|f\|^{2} \quad$ for $\quad f \in \mathscr{H}, t \in I$

(iii) $\left\|R_{D}(\lambda, \varphi)\right\| \leqq C_{I}$ for $\lambda \in S_{I}$.

(iv) $\sigma\left(H_{D}(\varphi)\right)=\sigma\left(H^{C_{1}}(\varphi)\right)+\ldots+\sigma\left(H^{C_{k}}(\varphi)\right)+e^{-2 i \varphi} R^{+}$.

Proof. We refer to [12 II], [13] and [14] for the proof, indicating only the main points, where [14] differs from [12 II] and [13]. One is, that (I5)-(I6) are simple consequences of (I3) by Lemma 2.7, and that (I7) follows from (I 1) and (I 2).

Another crucial estimate utilized in [14] in the proof of Lemma 4.1 will also be needed independently, so we formulate this separately as follows.

Lemma 4.2. For $|\operatorname{Im} \lambda| \geqq \varepsilon>0$,

(i) $\left\|v_{i j}(\varphi) R_{0}\left(e^{-2 i \varphi}(s+i t), \varphi\right)\right\| \leqq C_{\varepsilon} t^{-1 / 2}$

(ii) $\left\|v_{i j}^{2}(\varphi) R_{0}\left(e^{-2 i \varphi}(s+i t), \varphi\right)\right\| \leqq C_{\varepsilon} t^{-1 / 2}$.

Proof. Since $R_{0}\left(e^{-2 i \varphi}(s+i t), \varphi\right)=e^{2 i \varphi} R_{0}(s+i t)$, we can replace $R_{0}\left(e^{-2 i \varphi}(s+i t), \varphi\right)$ by $R_{0}(s+i t)$ in (i) and (ii). We have, setting $\lambda=s+i t$,

$\left\|q_{i j}(\varphi) R_{0}(\lambda)\right\| \leqq C\left\|R_{0}(\lambda)\right\| \leqq C|\operatorname{Im} \lambda|^{-1} \leqq C_{\varepsilon}|\operatorname{Im} \lambda|^{-1 / 2}$ for $|\operatorname{Im} \lambda| \geqq \varepsilon>0$.

The operator $u_{i j}(\varphi)$ is $H_{0}^{(i j)}$-smooth, that is

$$
\int_{-\infty}^{\infty}\left\|u_{i j}(\varphi) R_{0}^{(i j)}(s+i t) f\right\|^{2} d s \leqq C\|f\|^{2} \quad \text { for } \quad f \in \mathscr{H}, t \neq 0 .
$$


It is proved in [7] Theorem 5.1, that this implies

$$
\left\|u_{i j}(\varphi) R_{0}^{(i j)}(\lambda)\right\| \leqq C|\operatorname{Im} \lambda|^{-1 / 2} .
$$

By [13] Lemmas 4.1, 4.5, 4.6, 5.2, and Lemma 2.7 (see also 14 Lemma 4.1) we have

$$
u_{i j}(\varphi) R_{0}(\lambda) f=\int_{\Gamma} u_{i j}(\varphi) R_{0}^{(i j)}(\lambda-\mu) R_{0}^{\mathrm{rel}}(\mu) f d \mu
$$

where $\Gamma$ is the pair of parallel lines $\operatorname{Im} \mu=\mp \frac{1}{2} \operatorname{Im} \lambda$. Then for $f \in \mathscr{H}$,

$$
\left\|u_{i j}(\varphi) R_{0}(\lambda) f\right\|^{2} \leqq C|\operatorname{Im} \lambda|^{-1} \int_{\Gamma}\left\|R_{0}^{\mathrm{rel}}(\mu) f\right\|^{2} d \mu \leqq C|\operatorname{Im} \lambda|^{-1}\|f\|^{2},
$$

and (i) is proved.

The prove (ii), we notice that

$$
\left\|v_{i j}^{2}(\varphi) R_{0}(\lambda)\right\| \leqq C\left(\left\|u_{i j}^{2}(\varphi) R_{0}(\lambda)\right\|+\left\|u_{i j}(\varphi) R_{0}(\lambda)\right\|+\left\|R_{0}(\lambda)\right\|\right) .
$$

Since $u_{i j}^{2}(\varphi) \in L^{p}\left(R^{3}\right)$ for some $p>\frac{3}{2}, u_{i j}(\varphi)$ is $H_{0}$-smooth, and the proof of (i) applies.

From Lemmas 4.1 and 4.2 we obtain

Lemma 4.3. Let $D$ be a decomposition, and let $S_{I}(\varphi) \subset \varrho\left(H_{D}(\varphi)\right)$. Then for $\lambda \in S_{I}(\varphi)$ and $i<j=1, \ldots, n$ we have

(i) $\left\|v_{i j}(\varphi) R_{D}(\lambda, \varphi)\right\| \leqq C$

(ii) $\left\|\left(v_{i j}(\varphi)\right)^{2} R_{D}(\lambda, \varphi)\right\| \leqq C$.

Proof. By the second resolvent equation and Lemmas 2.6, 4.2(i), and 4.8(iii)

$$
\begin{aligned}
& \left\|v_{i j}(\varphi) R_{D}(\lambda, \varphi)\right\| \\
& =\left\|v_{i j}(\varphi) R_{0}(\lambda, \varphi)+\sum_{k<l=1}^{n} v_{i j}(\varphi) R_{0}(\lambda, \varphi) v_{k l}(\varphi)\right\| \cdot\left\|R_{D}(\lambda, \varphi)\right\| \text { for } \lambda \in S_{I}(\varphi) .
\end{aligned}
$$

Furthermore, by (i) and Lemma 4.2(ii)

$$
\begin{aligned}
& \left\|\left(v_{i j}(\varphi)\right)^{2} R_{D}(\lambda, \varphi)\right\|=\left\|\left(v_{i j}(\varphi)\right)^{2} R_{0}(\lambda, \varphi)+\left(v_{i j}(\varphi)\right)^{2} R_{0}(\lambda, \varphi) v_{k l}(\varphi) R_{D}(\lambda, \varphi)\right\| \\
& \leqq\left\|\left(v_{i j}(\varphi)\right)^{2} R_{0}(\lambda, \varphi)\right\|+\sum_{k<l=1}^{n}\left\|\left(v_{i j}(\varphi)\right)^{2} R_{0}(\lambda, \varphi)\right\| \\
& \| v_{k l}(\varphi)\left(R_{D}(\lambda, \varphi) \| \leqq C \quad \text { for } \quad \lambda \in S_{I}(\varphi) .\right.
\end{aligned}
$$

\section{Estimate of the Norm of $\left(I^{1}(\lambda, \varphi)\right)^{2}$}

We first apply the results of Sections 2 and 4 to establish some commutation estimates involving the operators $R_{D}(\lambda, \varphi)$.

Lemma 5.1. Let $D_{1}$ and $D_{2}$ be decompositions, and suppose that $C^{\prime} \cap C^{\prime \prime}=\emptyset$ for all clusters $C^{\prime}$ of $D_{1}$ and $C^{\prime \prime}$ of $D_{2}$. Let $\alpha>\frac{1}{2}, \beta<\frac{1}{2}$ be fixed, and let $S_{I}(\varphi) \subset \varrho\left(H_{D_{2}}(\varphi)\right)$. Then

$$
\begin{aligned}
& \left\|\left(1+r_{D_{1}}^{2}\right)^{-\alpha / 2} R_{D_{2}}(\lambda, \varphi) f\right\| \\
& \leqq C_{I}\left\|\left(1+r_{D_{1}}^{2}\right)^{-\beta / 4} R_{0}(\lambda, \varphi) f\right\| \text { for } \quad \lambda \in S_{I}(\varphi), f \in \mathscr{H} .
\end{aligned}
$$


Proof. (1) is equivalent to

$$
\left\|\left(1+r_{D_{1}}^{2}\right)^{-\alpha / 2} R_{D_{2}}(\lambda, \varphi)\left(H_{0}(\varphi)-\lambda\right)\left(1+r_{D_{1}}^{2}\right)^{\beta / 4}\right\| \leqq C_{I} \quad \text { for } \quad \lambda \in S_{I}(\varphi) .
$$

or to

$$
\left\|\left(1+r_{D_{1}}^{2}\right)^{-\alpha / 2} R_{D_{2}}(\lambda, \varphi) V_{D_{2}}(\varphi)\left(1+r_{D_{1}}^{2}\right)^{\beta / 4}\right\| \leqq C_{I} \quad \text { for } \quad \lambda \in S_{I}(\varphi) .
$$

Taking adjoints, we have

$$
\begin{aligned}
& \left\|\left(1+r_{D_{1}}^{2}\right)^{\beta / 4} V_{D_{2}}(-\varphi) R_{D_{2}}(\bar{\lambda},-\varphi)\left(1+r_{D_{1}}^{2}\right)^{-\alpha / 2}\right\| \\
& \leqq \\
& \quad \sqrt{2}\left\|\left(1+r_{D_{1}}^{2}\right)^{\beta / 2} R_{D_{2}}(\bar{\lambda},-\varphi)\left(1+r_{D_{1}}^{2}\right)^{-\alpha / 2}\right\| \\
& \quad+\sqrt{2}\left\|\left(V_{D_{2}}(-\varphi)\right)^{2} R_{D_{2}}(\bar{\lambda},-\varphi)\left(1+r_{D_{1}}^{2}\right)^{-\alpha / 2}\right\| .
\end{aligned}
$$

By Lemma 4.3 (ii), the second term in (4) is bounded for $\lambda \in S_{I}(\varphi)$. Taking adjoints, it remains to prove

$$
\left\|\left(1+r_{D_{1}}^{2}\right)^{-\alpha / 2} R_{D_{2}}(\lambda, \varphi)\left(1+r_{D_{1}}^{2}\right)^{\beta / 2}\right\| \leqq C \quad \text { for } \quad \lambda \in S_{I}(\varphi) .
$$

Let $S=C_{1} \cup C_{2}$, where $C_{1}$ contains all the clusters of $D_{1}$ and $C_{2}$ contains those of $D_{2}$.

By Lemma $4.1(2)$

$$
\int_{-\infty}^{\infty}\left\|R_{D_{2}}^{C_{2}}\left(e^{-2 i \varphi}(s+i t), \varphi\right) f\right\|^{2} d s \leqq C_{I}\|f\|^{2} \quad \text { for } \quad t \in I, f \in \mathscr{H},
$$

where

$$
H_{D_{2}}^{C_{2}}(\varphi)=I^{C_{1}} \otimes\left(H_{D_{2}}(\varphi)\right)_{\mathscr{H}_{C_{2}}} \otimes I_{\mathrm{rel}}^{C_{1}, C_{2}} .
$$

It follows from Lemma $2.2(4)$, that

$$
\left\|\left(1+r_{D_{1}}^{2}\right)^{-\alpha / 2} R_{0}^{C_{1}}(\lambda, \varphi)\left(1+r_{D_{1}}^{2}\right)^{\beta / 2} f\right\| \leqq C_{I}\left\|R_{0}^{C_{1}}(\lambda, \varphi) f\right\| \quad \text { for } \quad \lambda \in S_{I}(\varphi), f \in \mathscr{H},
$$

which in turn implies

$$
\begin{aligned}
& \int_{-\infty}^{\infty}\left\|\left(1+r_{D_{1}}^{2}\right)^{-\alpha / 2} R_{0}^{C_{1}}\left(e^{-2 i \varphi}(s+i t), \varphi\right)\left(1+r_{D_{1}}^{2}\right)^{\beta / 2} f\right\|^{2} d s \\
& \leqq C_{I}\|f\|^{2} \text { for } t \in I, f \in \mathscr{H} .
\end{aligned}
$$

From (6) and (8) it follows by [13] Lemma 5.2, see also [14] Lemma 4.1, that for some $\varepsilon>0$ and $f \in \mathscr{H}$

$$
\begin{aligned}
& \left(1+r_{D_{1}}^{2}\right)^{-\alpha / 2} R_{D_{2}}\left(e^{-2 i \varphi}(s+i t), \varphi\right)\left(1+r_{D_{1}}^{2}\right)^{\beta / 2} f \\
& =\int_{-\infty}^{\infty} R_{D_{2}}^{C_{2}}\left(e^{-2 i \varphi}(s-u+i(t-\varepsilon), \varphi)\right. \\
& \cdot\left[\left(1+r_{D_{1}}^{2}\right)^{-\alpha / 2} R_{0}^{C_{1}}\left(e^{-2 i \varphi}(u+i \varepsilon), \varphi\right)\left(1+r_{D_{1}}^{2}\right)^{\beta / 2}\right] f d u \\
& -\int_{-\infty}^{\infty} R_{D_{2}}^{C_{2}}\left(e^{-2 i \varphi}(s-u+i(t+\varepsilon), \varphi)\right. \\
& \quad \cdot\left[\left(1+r_{D_{1}}^{2}\right)^{-\alpha / 2} R_{0}^{C_{1}}\left(e^{-2 i \varphi}(u-i \varepsilon), \varphi\right)\left(1+r_{D_{1}}^{2}\right)^{\beta / 2}\right] f d u
\end{aligned}
$$


and hence

$$
\left\|\left(1+r_{D_{1}}^{2}\right)^{-\alpha / 2} R_{D_{2}}\left(e^{-2 i \varphi}(s+i t), \varphi\right)\left(1+r_{D_{1}}^{2}\right)^{\beta / 2}\right\| \leqq C \text { for } t \in I .
$$

Thus, (5) holds, and the Lemma is proved.

Lemma 5.2. Let $D$ and $D_{k}$ be decompositions, where $k$ is the number of clusters and single particles in $D_{k}$, and $S_{I}(\varphi) \subset \varrho\left(H_{D_{k}}(\varphi)\right)$. Let $A(\lambda)$ be a function from $S_{I}(\varphi)$ into $\mathscr{B}(\mathscr{H})$, such that

$$
\|A(\lambda)\| \leqq C \quad \text { for } \quad \lambda \in S_{I}(\varphi) .
$$

Let $\alpha>\frac{1}{2}, \beta<\frac{1}{2}$. Then there exists $\delta>0$, such that for $\lambda \in S_{I}(\varphi)$ and any $i, j$

$$
\left\|\left(1+r_{D}^{2}\right)^{-\alpha / 2} R_{D_{k}}(\lambda, \varphi) v_{i j}(\varphi) A(\lambda)\right\| \leqq C\left\|\left(1+r_{D}^{2}\right)^{-\beta / 2}\left(1+r_{i j}\right)^{-\varepsilon} A(\lambda)\right\|^{\delta} .
$$

Proof. We prove this for fixed $n$ by induction on $k$. For $k=n$ we have by Lemma 2.2 (4) and Lemma 2.6 (ii) for $\lambda \in S_{I}(\varphi), f \in \mathscr{H}$

$$
\begin{aligned}
& \left\|\left(1+r_{D}^{2}\right)^{-\alpha / 2} R_{0}(\lambda, \varphi) v_{i j}(\varphi) A(\lambda) f\right\| \\
& \leqq C\left\|R_{0}(\lambda, \varphi) w_{i j}(\varphi)\left(1+r_{D}^{2}\right)^{-\beta / 2}\left(1+r_{i j} e^{i \varphi}\right)^{-\varepsilon} A(\lambda) f\right\| \\
& \leqq C\left\|\left(1+r_{D}^{2}\right)^{-\beta / 2}\left(1+r_{i j}\right)^{-\varepsilon} A(\lambda) f\right\|,
\end{aligned}
$$

and (9) holds with $\delta=1$.

In the following estimates $\delta>0$ denotes different constants, fixed in each estimate. We now make the induction assumption

$\left(^{*}\right) \quad$ The estimate (9) holdes for all $A(\lambda), i, j, D$, and $D_{m}$ with $m \geqq k+1$.

Let us verify (9) for $m=k$. Using Equation (3) of Section 4 and (*) we obtain for some $\delta>0$.

$$
\begin{aligned}
& \left\|\left(1+r_{D}^{2}\right)^{-\alpha / 2} R_{D_{k}}(\lambda, \varphi) v_{i j}(\varphi) A(\lambda)\right\| \\
& \leqq C \sum_{D_{m} \subset D_{k}}\left\|\left(1+r_{D}^{2}\right)^{-\alpha / 2} R_{D_{m}}(\lambda, \varphi) v_{i j}(\varphi) A(\lambda)\right\| \\
& +\left\|\left(1+r_{D}^{2}\right)^{-\alpha / 2} I_{D_{k}}^{l}(\lambda, \varphi) R_{D_{k}}(\lambda, \varphi) v_{i j}(\varphi) A(\lambda)\right\| \\
& \leqq C\left\|\left(1+r_{D}^{2}\right)^{-\beta / 2}\left(1+r_{i j}\right)^{-\varepsilon} A(\lambda)\right\|^{\delta}+\left\|\left(1+r_{D}^{2}\right)^{-\alpha / 2} I_{D_{k}}^{l}(\lambda, \varphi) R_{D_{k}}(\lambda, \varphi) v_{i j}(\varphi) A(\lambda)\right\| .
\end{aligned}
$$

We estimate a typical term arising from $I_{D_{k}}^{l}(\lambda, \varphi)$ using successively Lemma 2.2(4), Lemma 2.6 (ii), Lemma 1.1(2), (3), Lemma 2.4, and Lemma 4.3 (i), and obtain for some $\delta>0$

$$
\begin{aligned}
& \left\|\left(1+r_{D}^{2}\right)^{-\alpha / 2} R_{0}(\lambda, \varphi) v_{\alpha_{1}}(\varphi) R_{D_{n-1}}(\lambda, \varphi) v_{\alpha_{2}}(\varphi) \ldots v_{\alpha_{n-k}}(\varphi) R_{D_{k}}(\lambda, \varphi) v_{i j}(\varphi) A(\lambda)\right\| \\
\leqq & C \| R_{0}(\lambda, \varphi)\left(1+r_{D}^{2}\right)^{-\beta / 2} w_{\alpha_{1}}(\varphi)\left(1+r_{\alpha_{1}}^{2}\right)^{-\varepsilon} R_{D_{n-1}}(\lambda, \varphi) \\
& v_{\alpha_{2}}(\varphi) \ldots v_{\alpha_{n-k}}(\varphi) R_{D_{k}}(\lambda, \varphi) v_{i j}(\varphi) A(\lambda) \| \\
\leqq & C\left\|\left(1+r_{D}^{2}\right)^{-\beta / 2}\left(1+r_{\alpha_{1}}^{2}\right)^{-\varepsilon} R_{D_{n-1}}(\lambda, \varphi) v_{\alpha_{2}}(\varphi) \ldots v_{\alpha_{n-k}}(\varphi) R_{D_{k}}(\lambda, \varphi) v_{i j}(\varphi) A(\lambda)\right\| \\
\leqq & C\left\|\left(1+r_{D^{1}}^{2}\right)^{-\varepsilon} R_{D_{n-1}}(\lambda, \varphi) v_{\alpha_{2}}(\varphi) \ldots v_{\alpha_{n-k}}(\varphi) R_{D_{k}}(\lambda, \varphi) v_{i j}(\varphi) A(\lambda)\right\| \\
\leqq & C\left\|\left(1+r_{D^{1}}^{2}\right)^{-\alpha / 2} R_{D_{n-1}}(\lambda, \varphi) v_{\alpha_{2}}(\varphi) \ldots v_{\alpha_{n-k}}(\varphi) R_{D_{k}}(\lambda, \varphi) v_{i j}(\varphi) A(\lambda)\right\|^{\delta}
\end{aligned}
$$

where $D^{1}$ is the decomposition obtained from $D$ by connecting the pair $\alpha_{1}$. 
The last norm in the chain of inequalities (11) is estimated in the same way using successively (*), Lemma 4.3 (i), Lemma 1.1 (2), (3), Lemma 2.4, and Lemma 4.3 (i), and we obtain for some $\delta>0$

$$
\begin{aligned}
& \left\|\left(1+r_{D^{1}}^{2}\right)^{-\alpha / 2} R_{D_{n-1}}(\lambda, \varphi) v_{\alpha_{2}}(\varphi) \ldots v_{\alpha_{n-k}}(\varphi) R_{D_{k}}(\lambda, \varphi) v_{i j}(\varphi) A(\lambda)\right\| \\
& \leqq C\left\|\left(1+r_{D^{2}}\right)^{-\alpha / 2} R_{D_{n-2}}(\lambda, \varphi) v_{\alpha_{3}}(\varphi) \ldots v_{\alpha_{n-k}}(\varphi) R_{D_{k}}(\lambda, \varphi) v_{i j}(\varphi) A(\lambda)\right\|^{\delta}
\end{aligned}
$$

where $D^{2}$ is the decomposition obtained from $D^{1}$ by joining the pair $\alpha_{2}$.

We continue in this way and obtain after $n-k$ steps for some $\delta>0$ the estimate

$$
\begin{aligned}
& \left\|\left(1+r_{D}^{2}\right)^{-\alpha / 2} R_{0}(\lambda, \varphi) v_{\alpha_{1}}(\varphi) R_{D_{n-1}}(\lambda, \varphi) v_{\alpha_{2}}(\varphi) \ldots v_{\alpha_{n-k}}(\varphi) R_{D_{k}}(\lambda, \varphi) v_{i j}(\varphi) A(\lambda)\right\| \\
& \leqq C\left\|\left(1+r_{D^{k}}^{2}\right)^{-\alpha / 2} R_{D_{k}}(\lambda, \varphi) v_{i j}(\varphi) A(\lambda)\right\|^{\delta}
\end{aligned}
$$

where $D^{k}$ is the decomposition obtained by connecting all particles which are connected in either $D$ or $D_{k}$.

We now use Equation (4) of Section 4 to estimate the norm on the right hand side of (12). This yields

$$
\begin{aligned}
& \left\|\left(1+r_{D^{k}}^{2}\right)^{-\alpha / 2} R_{D_{k}}(\lambda, \varphi) v_{i j}(\varphi) A(\lambda)\right\| \\
& \leqq C \sum_{D_{m} \subset D_{k}}\left\|\left(1+r_{D_{k}}^{2}\right)^{-\alpha / 2} R_{D_{m}}(\lambda, \varphi) v_{i j}(\varphi) A(\lambda)\right\| \\
& \quad+\left\|\left(1+r_{D^{k}}^{2}\right)^{-\alpha / 2} R_{D_{k}}(\lambda, \varphi) I_{D_{k}}^{r}(\lambda, \varphi) v_{i j}(\varphi) A(\lambda)\right\| .
\end{aligned}
$$

The first terms in (13) are estimated as the first terms in (10), using $\left(^{*}\right)$ and Lemma $1.1(1)$, for some $\delta>0$ by

$$
\begin{aligned}
& \left\|\left(1+r_{D^{k}}^{2}\right)^{-\alpha / 2} R_{D_{m}}(\lambda, \varphi) v_{i j}(\varphi) A(\lambda)\right\| \leqq C\left\|\left(1+r_{D^{k}}^{2}\right)^{-\beta / 2}\left(1+r_{i j}\right)^{-\varepsilon} A(\lambda)\right\|^{\delta} \\
& \leqq\left\|\left(1+r_{D}^{2}\right)^{-\beta / 2}\left(1+r_{i j}\right)^{-\varepsilon} A(\lambda)\right\|^{\delta} .
\end{aligned}
$$

The last term on the right hand side of (13) is estimated using Lemma 1.1 (1) by

$$
\begin{aligned}
& \left\|\left(1+r_{D^{k}}^{2}\right)^{-\alpha / 2} R_{D_{k}}(\lambda, \varphi) I_{D_{k}}^{r}(\lambda, \varphi) v_{i j}(\varphi) A(\lambda)\right\| \\
& \leqq\left\|\left(1+r_{D^{0}}^{2}\right)^{-\alpha / 2} R_{D_{k}}(\lambda, \varphi) I_{D_{k}}^{r}(\lambda, \varphi) v_{i j}(\varphi) A(\lambda)\right\|,
\end{aligned}
$$

where $D^{0}$ is the decomposition obtained by connecting the pairs which are connected in $D$, but not in $D_{k}$.

We now apply Lemma 5.1, Lemma 2.4, Lemma 4.3 (i), and Lemma 2.2 (4) to obtain for some $\delta>0$ the estimate

$$
\begin{aligned}
& \left\|\left(1+r_{D^{0}}^{2}\right)^{-\alpha / 2} R_{D_{k}}(\lambda, \varphi) I_{D_{k}}^{r}(\lambda, \varphi) v_{i j}(\varphi) A(\lambda)\right\| \\
& \leqq C\left\|\left(1+r_{D^{0}}^{2}\right)^{-\beta / 4} R_{0}(\lambda, \varphi) I_{D_{k}}^{r}(\lambda, \varphi) v_{i j}(\varphi) A(\lambda)\right\| \\
& \leqq C\left\|\left(1+r_{D^{0}}^{2}\right)^{-\alpha / 2} R_{0}(\lambda, \varphi) I_{D_{k}}^{r}(\lambda, \varphi) v_{i j}(\varphi) A(\lambda)\right\|^{\delta} \\
& \leqq C\left\|R_{0}(\lambda, \varphi)\left(1+r_{D^{0}}^{2}\right)^{-\beta / 2} I_{D_{k}}^{r}(\lambda, \varphi) v_{i j}(\varphi) A(\lambda)\right\|^{\delta} .
\end{aligned}
$$

A typical term of the operator, whose norm is the last one in the chain of inequalities (16), is estimated, using Lemma 2.6 (ii), Lemma 1.1 (2), (3), Lemma 2.4, 
and Lemma 4.3 (i), for some $\delta>0$ by

$$
\begin{aligned}
& \| R_{0}(\lambda, \varphi)\left(1+r_{D^{0}}^{2}\right)^{-\beta / 2} V_{\alpha_{n-k}}(\varphi) R_{D_{k+1}}(\lambda, \varphi) v_{\alpha_{n-k-1}}(\varphi) \ldots \\
& \ldots v_{\alpha_{2}}(\varphi) R_{D_{n-1}}(\lambda, \varphi) v_{\alpha_{1}}(\varphi) R_{0}(\lambda, \varphi) v_{i j}(\varphi) A(\lambda) \| \\
& \leqq C \|\left(1+r_{D^{0}}^{2}\right)^{-\beta / 2}\left(1+r_{\alpha_{n-k}}\right)^{-\varepsilon} R_{D_{k+1}}(\lambda, \varphi) v_{\alpha_{n-k-1}}(\varphi) \ldots \\
& \quad \ldots v_{\alpha_{2}}(\varphi) R_{D_{n-1}}(\lambda, \varphi) v_{\alpha_{1}}(\varphi) R_{0}(\lambda, \varphi) v_{i j}(\varphi) A(\lambda) \| \\
& \leqq C \|\left(1+r_{D^{(1)}}^{2}\right)^{-\varepsilon} R_{D_{k+1}}(\lambda, \varphi) v_{\alpha_{n-k-1}}(\varphi) \ldots \\
& \quad \ldots v_{\alpha_{2}}(\varphi) R_{D_{n-1}}(\lambda, \varphi) v_{\alpha_{1}}(\varphi) R_{0}(\lambda, \varphi) v_{i j}(\varphi) A(\lambda) \| \\
& \leqq C \|\left(1+r_{D^{(1)}}^{2}\right)^{-\alpha / 2} R_{D_{k-1}}(\lambda, \varphi) v_{\alpha_{n-k-1}}(\varphi) \ldots \\
& \quad \ldots v_{\alpha_{2}}(\varphi) R_{D_{n-1}}(\lambda, \varphi) v_{\alpha_{1}}(\varphi) R_{0}(\lambda, \varphi) v_{i j}(\varphi) A(\lambda) \|^{\delta}
\end{aligned}
$$

where $D^{(1)}$ is obtained from $D^{0}$ by connecting the pair $\alpha_{n-k}$. We estimate the last term in the chain of inequalities (17) in the same way, using $\left(^{*}\right)$, Lemma $4.3(\mathrm{i})$, Lemma 1.1 (2), (3), Lemma 2.4, and Lemma 4.3 (i), and obtain for some $\delta>0$

$$
\begin{aligned}
& \|\left(1+r_{D^{(1)}}^{2}\right)^{-\alpha / 2} R_{D_{k+1}}(\lambda, \varphi) v_{\alpha_{n-k-1}}(\varphi) \ldots \\
& \quad \ldots v_{\alpha_{2}}(\varphi) R_{D_{n-1}}(\lambda, \varphi) v_{\alpha_{1}}(\varphi) R_{0}(\lambda, \varphi) v_{i j}(\varphi) A(\lambda) \| \\
& \leqq C \|\left(1+r_{\left.D^{(2)}\right)}^{2}\right)^{-\alpha / 2} R_{D_{k+2}}(\lambda, \varphi) v_{\alpha_{n-k-2}}(\varphi) \ldots \\
& \quad \ldots v_{\alpha_{2}}(\varphi) R_{D_{n-1}}(\lambda, \varphi) v_{\alpha_{1}}(\varphi) R_{0}(\lambda, \varphi) v_{i j}(\varphi) A(\lambda) \|^{\delta}
\end{aligned}
$$

where $D^{(2)}$ is obtained from $D^{(1)}$ by connecting the pair $\alpha_{n-k-1}$. Repeating this process, we obtain after $n-k$ steps for some $\delta>0$

$$
\begin{aligned}
& \left(R_{0}(\lambda, \varphi)\left(1+r_{D^{0}}^{2}\right)^{-\beta / 2} v_{\alpha_{n-k}}(\varphi) R_{D_{k+1}}(\lambda, \varphi) v_{\alpha_{n-k-1}}(\varphi) \ldots\right. \\
& \quad \ldots v_{\alpha_{2}}(\varphi) R_{D_{n-1}}(\lambda, \varphi) v_{\alpha_{1}}(\varphi) R_{0}(\lambda, \varphi) v_{i j}(\varphi) A(\lambda) \| \\
& \leqq C\left\|\left(1+r_{D^{(k)}}^{2}\right)^{-\alpha / 2} R_{0}(\lambda, \varphi) v_{i j}(\varphi) A(\lambda)\right\|^{\delta} .
\end{aligned}
$$

Here $D^{(k)}$ is obtained from $D^{0}$ by connecting all pairs of $D_{k}$. We notice that $D^{(k)}$ is also obtained from $D$ by connecting all pairs of $D_{k}$, that is $D^{(k)}=D^{k}$. Using this and $(*)$, Lemma 2.6 (i) and Lemma 2.6 (ii) we obtain finally for some $\delta>0$

$$
\begin{aligned}
& \left\|\left(1+r_{D^{(k)}}^{2}\right)^{-\alpha / 2} R_{0}(\lambda, \varphi) v_{i j}(\varphi) A(\lambda)\right\| \\
& \leqq C\left\|\left(1+r_{D}^{2}\right)^{-\beta / 2}\left(1+r_{i j}\right)^{-\varepsilon} A(\lambda)\right\|^{\delta}
\end{aligned}
$$

Combining the estimates (10), (12)-(16), (19), and (20), we obtain (9) for the decomposition $D_{k}$; by induction (9) holds for $2 \leqq k \leqq n$, and the Lemma is proved.

Lemma 5.3. Let $S_{I} \subset \bigcap_{D} \varrho\left(H_{D}(\varphi)\right)$. Then there exists $\delta>0$ such that

$$
\left\|\left(I^{l}\left(e^{-2 i \varphi}(s+i t), \varphi\right)\right)^{2}\right\| \leqq C S^{-\delta} \quad \text { for } \quad s \in R, t \in I .
$$

Proof. A typical term of $\left(I^{l}\left(e^{-2 i \varphi}(s+i t), \varphi\right)\right)^{2}$ is estimated, using Lemma $2.6(\mathrm{i})$ and Lemma 4.3 (i) by

$$
\begin{aligned}
& \left\|R_{0}(\lambda, \varphi) v_{\alpha_{1}}(\varphi) \ldots R_{D_{2}}(\lambda, \varphi) v_{\alpha_{n-1}}(\varphi) R_{0}(\lambda, \varphi) v_{\beta_{1}}(\varphi) \ldots R_{D_{2}}(\lambda, \varphi) v_{\beta_{n-1}}(\varphi)\right\| \\
& \leqq C\left\|R_{0}(\lambda, \varphi) v_{\alpha_{1}}(\varphi) \ldots R_{D_{2}}(\lambda, \varphi) v_{\alpha_{n-1}}(\varphi) R_{0}(\lambda, \varphi)\right\| .
\end{aligned}
$$


The operator on the right hand side of (22) is estimated using Lemma 2.6 (ii), Lemma 2.4, and Lemma 4.3 (i) for some $\delta>0$ by

$$
\begin{aligned}
& \left\|R_{0}(\lambda, \varphi) v_{\alpha_{1}}(\varphi) \ldots R_{D_{2}}(\lambda, \varphi) v_{\alpha_{n-1}}(\varphi) R_{0}(\lambda, \varphi)\right\| \\
& \leqq C\left\|\left(1+r_{\alpha_{1}}^{2}\right)^{-\alpha / 2} R_{D_{n-1}}(\lambda, \varphi) v_{\alpha_{2}}(\varphi) \ldots R_{D_{2}}(\lambda, \varphi) v_{\alpha_{n-1}}(\varphi) R_{0}(\lambda, \varphi)\right\|^{\delta} .
\end{aligned}
$$

The operator on the right hand side of (23) is estimated using Lemma 5.2, Lemma 4.3 (i), Lemma 1.1 (2), and Lemma 2.4 for some $\delta>0$ by

$$
\begin{aligned}
& \left\|\left(1+r_{\alpha_{1}}^{2}\right)^{-\alpha / 2} R_{D_{n-1}}(\lambda, \varphi) v_{\alpha_{2}}(\varphi) \ldots R_{D_{2}}(\lambda, \varphi) v_{\alpha_{n-1}}(\varphi) R_{0}(\lambda, \varphi)\right\| \\
& \leqq C\left\|\left(1+r_{D_{n-2}}^{2}\right)^{-\alpha / 2} R_{D_{n-2}}(\lambda, \varphi) v_{\alpha_{3}}(\varphi) \ldots R_{D_{2}}(\lambda, \varphi) v_{\alpha_{n-1}}(\varphi) R_{0}(\lambda, \varphi)\right\|^{\delta} .
\end{aligned}
$$

Repeating this process, we obtain after $n-1$ steps for some $\delta>0$

$$
\left\|R_{0}(\lambda, \varphi) v_{\alpha_{1}}(\varphi) \ldots R_{D_{2}}(\lambda, \varphi) v_{\alpha_{n-1}}(\varphi) R_{0}(\lambda, \varphi)\right\| \leqq C\left\|\left(1+r_{S}^{2}\right)^{-\alpha / 2} R_{0}(\lambda, \varphi)\right\|^{\delta} .
$$

Combining (25) with Lemma 2.5 and summing over all the terms of $(I(\lambda, \varphi))^{2}$, we obtain (21), and the Lemma is proved.

\section{Decomposition of the Operator $\boldsymbol{H}(\varphi)$}

The basic properties of $R(\varphi)$ can now be established, verifying the induction assumption of Section 4 and proving the estimates required for the decomposition.

Theorem 6.1. The operator $H(\varphi)$ has the properties (I1)-(I7) listed in the induction assumption of Section 4 for the operators $H^{C}(\varphi)$. In particular we have for $S_{I}(\varphi) \subset$ $\varrho(H(\varphi)), t \in I$ and $f \in \mathscr{H}$

$$
\int_{-\infty}^{\infty}\left\|R\left(e^{-2 i \varphi}(s+i t), \varphi\right) f\right\|^{2} d s \leqq C_{I}\|f\|^{2} .
$$

Proof. It suffices to consider (I1)-(I3), since (I4)-(I7) follow from these. By Lemma 4.1, the operators $I^{l}(\lambda, \varphi)$ and $Q(\lambda, \varphi)=\sum_{m=2 D_{m}}^{n}(-1)^{m-1}(m-1) ! R_{D_{m}}(\lambda, \varphi)$ are analytic for $\lambda \in \bigcap_{D} \varrho\left(H_{D}(\varphi)\right)=\mathbb{C} \backslash\left\{\mu(\varphi)+e^{-2 i \varphi} R^{+} \mid \mu(\varphi) \in \Sigma(\varphi)\right\}$.

Then (I1) and the first two statements of (I2) for the operator $H(\varphi)$ follow as in [3]. In the same way it follows, that by iteration of Equation (3) of Section 4 we obtain

$$
R(\lambda, \varphi)=\left(1-\left(I^{l}(\lambda, \varphi)\right)^{2}\right)^{-1}\left(1+I^{l}(\lambda, \varphi)\right) Q(\lambda, \varphi),
$$

where $\left(1-\left(I^{l}(\lambda, \varphi)\right)^{2}\right)^{-1}$ is meromorphic on $\mathbb{C} \backslash \sigma_{e}(H(\varphi))$ with a set of poles containing and having the same properties as the set of poles of $\left(1-I^{l}(\lambda, \varphi)\right)^{-1}$.

By Lemma 5.3

$$
\left\|\left(I^{l}\left(e^{-2 i \varphi}(s+i t), \varphi\right)\right)^{2}\right\| \underset{s \rightarrow \infty}{\longrightarrow} \infty
$$

uniformly for $t \in I$, provided that $S_{I}(\varphi) \subset \cdot \bigcap_{D} \varrho\left(H_{D}(\varphi)\right)$. 
This implies the last statement of (I2), and moreover we obtain for some $K>0$ the estimate

$$
\begin{aligned}
& \left\|R\left(e^{-2 i \varphi}(s+i t), \varphi\right) f\right\| \\
& \leqq C\left\|Q\left(e^{-2 i \varphi}(s+i t), \varphi\right) f\right\| \text { for } f \in \mathscr{H} \text { and } s \geqq K, t \in I .
\end{aligned}
$$

Now we obtain by the second resolvent equation and Lemma 4.3 (i) for $\lambda \in S_{I}(\varphi), f \in \mathscr{H}$

$$
\begin{aligned}
& \|Q(\lambda, \varphi) f\| \leqq C \sum_{D}\left\|R_{D}(\lambda, \varphi) f\right\| \\
& \leqq C \sum_{D}\left\{\left\|R_{0}(\lambda, \varphi) f\right\|+\sum_{\alpha \in D}\left\|R_{D}(\lambda, \varphi) v_{\alpha}(\varphi)\right\|\left\|R_{0}(\lambda, \varphi) f\right\|\right\} \leqq C\left\|R_{0}(\lambda, \varphi) f\right\| .
\end{aligned}
$$

From (3) and (4) follows

$$
\int_{K}^{\infty}\left\|R\left(e^{-2 i \varphi}(s+i t), \varphi\right) f\right\|^{2} d s \leqq C\|f\|^{2}, \quad f \in \mathscr{H}, t \in I .
$$

This implies (1) which is (I3) with $H_{C}(\varphi)$ replaced by $H(\varphi)$.

Remark ([13] Remark 3.5). From the fact that $\|R(\lambda, \varphi)\| \leqq C$ for $\lambda \in S_{I}(\varphi)$ it follows that for $f \in \mathscr{H}, R\left(e^{-2 i \varphi}(s+i t), \varphi\right) f \underset{s \rightarrow \infty}{\longrightarrow} 0$ uniformly for $t \in I$.

Now the decomposition theorem for $H(\varphi)$ follows from Theorem 6.1 as shown by van Winter ([13] Theorem 8.1).

Theorem 6.2. Let $\Gamma$ be a pair of oppositely oriented lines $\Lambda_{1}=\left\{e^{-2 i \varphi}\left(s+i t_{1}\right) \mid \infty>\right.$ $s>-\infty\}$ and $\Lambda_{2}=\left\{e^{-2 i \varphi}\left(s+i t_{2}\right) \mid-\infty<s<\infty\right\}$, where $t_{1}<t_{2}$, such that $\Lambda_{1}$ and $\Lambda_{2}$ are contained in $\varrho(\varphi)$, and such that at least one half-line $\mu(\varphi)+e^{-2 i \varphi} R^{+}$of $\sigma_{e}(\varphi)$ lies between $\Lambda_{1}$ and $\Lambda_{2}$. Then a bounded linear operator $P_{\Gamma}(\varphi)$ is defined by the expression

$$
\left(f, P_{\Gamma}(\varphi) g\right)=\frac{1}{2 \pi \mathrm{i}} \lim _{K \rightarrow \infty} \int_{\Gamma_{K}}(\mathrm{f}, \mathrm{R}(\lambda, \varphi) \mathrm{g}) \mathrm{d} \lambda, \quad \mathrm{f}, \mathrm{g} \in \mathscr{H},
$$

where $\Gamma_{K}=\{\lambda \in \Gamma|| s \mid \leqq K\}$.

The operator $P_{\Gamma}(\varphi)$ is a projection operator, which is $\Gamma$-independent as long as $\Gamma$ does not cross $\sigma(\varphi)$ and satisfies

(i) $P_{\Gamma}(\varphi) R(\lambda, \varphi)=R(\lambda, \varphi) P_{\Gamma}(\varphi)$ for $\lambda \in \varrho(\varphi)$.

(ii) The operator $R_{\Gamma}(\lambda, \varphi)=P_{\Gamma}(\varphi) R(\lambda, \varphi)$ has an analytic continuation to the region outside of $\Gamma$ given by

$$
\left(f, R_{\Gamma}(\lambda, \varphi) g\right)=\frac{1}{2 \pi i} \lim _{K \rightarrow \infty} \int_{\Gamma_{K}}(f, R(\mu, \varphi) g)(\mu-\lambda)^{-1} d \mu .
$$

(iii) If $\Gamma^{\prime}$ and $\Gamma^{\prime \prime}$ are contours such that $\left[t_{1}^{\prime}, t_{2}^{\prime}\right] \cap\left[t_{1}^{\prime \prime}, t_{2}^{\prime \prime}\right]=\emptyset$, then $P_{\Gamma^{\prime}}(\varphi) P_{\Gamma^{\prime \prime}}(\varphi)=P_{\Gamma^{\prime \prime}}(\varphi) P_{\Gamma^{\prime}}(\varphi)=0$.

(iv) If $\sigma(\varphi)$ is contained between $\Lambda_{1}$ and $\Lambda_{2}$, then

$$
P_{\Gamma}(\varphi)=I \text {. }
$$


Remark. Theorems 6.1 and 6.2 are valid also if there is an infinite number of thresholds, since even in that case $\sigma_{e}(H(\varphi))$ has the relatively simple structure described in $I$ (1) and $I$ (2). This allows the convolution representation of section 4 with a finite number of parallel lines, and consequently the estimates of Section 5. If there is a finite number of thresholds, resonances, and eigenvalues, we obtain a complete decomposition of $H(\varphi)$ on subspaces corresponding to the cuts, resonances and eigenvalues. If the number is infinite, the question remains whether the sum of individual projections converges. In this case Theorem 6.2 allows a decomposition of $H(\varphi)$ as a sum of operators each of which has as its spectrum one cut with a cluster of resonances or a cluster of cuts with a cluster of resonances. In the 3-body case for example only the first type would occur except possibly at 0 .

Acknowledgement. I wish to express my thanks to J. Ginibre for the communication of the unpublished proof of Lemma 2.3.

I also want to thank the Department of Mathematics, University of Virginia, for hospitality in January-February 1976, when this paper was concluded with the support of NSF grant MPS-08208 sponsored by UCLA and a travel grant from the University of Virginia.

\section{References}

1. Agmon, S.: Spectral properties of Schrödinger operators and scattering theory. Ann. Scuola Norm. Sup. Pisa, Clas. Sci. Ser. IV II, 151-218 (1975)

2. Babbitt,D., Balslev,E.: A characterisation of dilation-analytic potentials and vectors. J. Funct. Anal. 18, 1-14 (1975)

3. Balslev, E., Combes, J. M.: Spectral properties of many-body Schrödinger operators with dilationanalytic interactions. Commun. math. Phys. 22, 280-294 (1971)

4. Faddeev, L.D.: Mathematical aspects of the three-body problem in the quantum scattering theory. Israel program for scientific translations, Jerusalem 1975

5. Ginibre, J., Moulin, M.: Hilbert space approach to the quantum-mechanical three-body problem. Ann. Inst. Poincaré, Section A (Physique Théorique) XXI, 97-145 (1974)

6. Iorio, R.J., O’Carroll, M.: Asymptotic completeness for multiparticle Schrödinger Hamiltonians with weak potentials. Commun. math. Phys. 27, 137-145 (1972)

7. Kato, T.: Wave operators and similarity for some non-selfadjoint operators. Math. Ann. 162, $258-279(1966)$

8. Paley, R., Wiener, N.: Fourier transforms in the complex domain, A.M.S. 1934

9. Sigal,I.: Mathematical foundations of quantum mechanical scattering theory for multiparticle systems. Preprint Tel-Aviv 1975

10. Simon, B.: Quadratic form techniques and the Balslev-Combes theorem. Commun. math. Phys. 27, 1-12 (1972)

11. Thomas, L. E.: Asymptotic completeness in two- and three-particle quantum mechanical scattering. Ann. Phys. 90, 127-165 (1975)

12. van Winter, C.: Complex dynamical variables for multiparticle systems with analytic interactions I-II. J. Math. Anal. Appl. 47, 633-670 (1974); 48, 368-399 (1974)

13. van Winter,C.: Invariant subspaces of analytic multiparticle Hamiltonians. J. Math. Anal. Appl. 49, 88-123 (1975)

14. Balslev,E.: Decomposition of many-body Schrödinger operators, University of Aarhus, March 1976

Communicated by W. Hunziker

Received August 9, 1976 